\title{
$B$ and $B_{c}$ mesons with NRQCD and Clover actions
}

\author{
Presented by A. Ali Khan ${ }^{\mathrm{a}}$ and T. Bhattacharya ${ }^{\mathrm{b}} *$ \\ ${ }^{a}$ Department of Physics and Astronomy, University of Glasgow, Glasgow, G12 8QQ, Scotland. \\ bT-8 Group, MS B285, Los Alamos National Laboratory, Los Alamos, New Mexico 87545, U.S.A.
}

We present preliminary results from our study of the heavy-light spectrum and decay constants. For the heavy quark, we use NRQCD at various masses around and above the $b$ quark mass. For the first time, the heavy quark action and the heavy-light current consistently include corrections at second order in the non-relativistic expansion, as well as the leading finite $a$ corrections. The light quarks are simulated using a tadpole-improved Clover action at various masses in the strange and $c$ quark region.

\section{INTRODUCTION}

Heavy-light hadrons are of topical interest in theoretical and experimental particle physics. The $B$ meson decay constant $f_{B}$ is required, together with the bag parameter $B_{B}$, for a determination of the poorly known elements of the CKM matrix, in particular, of the phase leading to CPviolation in nature. Comparison with experimental results on the heavy-light spectrum available currently and in the near future provides an important test of the reliablity of the approximations used in these calculations.

Using NRQCD, it is possible to simulate the $b$ quark directly, without having to use extrapolations in the heavy quark mass. Earlier results for $f \sqrt{M}$, obtained using NRQCD consistent through $O\left(1 / m_{Q}^{0}\right)$ where $m_{Q}^{0}$ is the bare heavy quark mass (see e.g. 迎2), showed that $f \sqrt{M}$ has a large slope as a function of $1 / M$. Such a behavior would be an indication that higher order terms are important. We address this issue here.

\section{THIS SIMULATION}

In this study, the heavy quark action and heavy-light currents are consistently improved through $O\left(1 /\left(m_{Q}^{0}\right)^{2}\right)$ at tree level. We also include the leading $O\left(a^{2}\right)$ lattice spacing correction to the lattice laplacian, and a term to compen-

\footnotetext{
*In collaboration with S. Collins and C. T. H. Davies, (Glasgow Univ., UKQCD Collab.), R. Gupta (LANL), J. Shigemitsu (Ohio State Univ.) and J. Sloan (SCRI).
}

sate for the leading $O(a)$ error in the discretization of the temporal derivative. Furthermore, we include the leading relativistic correction, which is expected to be the largest $O\left(1 /\left(m_{Q}^{0}\right)^{3}\right)$ correction. This action is also consistent through $O\left(v^{4}\right)$ in the heavy quark velocity in heavy-heavy systems.

We calculate the heavy quark propagator $G_{t}$ on a timeslice $t$ using the explicitly time-reversal invariant evolution equation:

$$
\begin{aligned}
& G_{t+1}=\left(1-\frac{\delta^{-} H}{2}\right)\left(1-\frac{H_{0}}{2 n}\right)^{n} \\
& U_{4}^{\dagger}\left(1-\frac{H_{0}}{2 n}\right)^{n}\left(1-\frac{\delta^{+} H}{2}\right) G_{t} .
\end{aligned}
$$

The free heavy quark Hamiltonian $H_{0}$ contains only the kinetic energy term

$H_{0}=-\frac{\Delta^{(2)}}{2 m_{Q}^{0}}$,

and the other terms are similar to [3]:

$$
\begin{aligned}
\delta^{ \pm} H=- & \frac{g \vec{\sigma} \cdot \vec{B}}{2 m_{Q}^{0}}+\frac{i g}{8\left(m_{Q}^{0}\right)^{2}}\left(\vec{\Delta} \cdot \overrightarrow{E^{ \pm}}-\overrightarrow{E^{ \pm}} \cdot \vec{\Delta}\right) \\
& -\frac{g}{8\left(m_{Q}^{0}\right)^{2}} \vec{\sigma} \times\left(\vec{\Delta} \times \overrightarrow{E^{ \pm}}-\overrightarrow{E^{ \pm}} \times \vec{\Delta}\right) \\
& -\frac{\left(\Delta^{(2)}\right)^{2}}{8\left(m_{Q}^{0}\right)^{3}}+\frac{a^{2} \Delta^{(4)}}{24 m_{Q}^{0}}-\frac{a\left(\Delta^{(2)}\right)^{2}}{16 n\left(m_{Q}^{0}\right)^{2}} .
\end{aligned}
$$

Here $\vec{\Delta}, \Delta^{(2)}$ and $\Delta^{(4)}$ denote the tadpoleimproved symmetric lattice discretization of the 
spatial covariant derivative, the laplacian and the lattice-artifact fourth derivative described in [3]. The temporal gauge link, the magnetic field $B$ and the electric field $E$ are also tadpole-improved (i.e. each link is divided by $\left.u_{0}=0.878\right) . E^{+}$and $E^{-}$denote the traceless forward and backward 2-leaf clover electric fields, respectively.

The heavy light currents $(J)$ are also improved to the same order:

$J=J_{0}+\frac{1}{2 m_{Q}^{0}} J_{1}+\frac{1}{8\left(m_{Q}^{0}\right)^{2}} J_{2}$.

where, at tree level,

$$
\begin{array}{r}
J_{0}=\bar{q} \Gamma Q, \quad J_{1}=\bar{q} \Gamma(\vec{\gamma} \cdot \vec{\Delta}) Q, \\
J_{2}=\bar{q} \Gamma\left(\Delta^{(2)}+\vec{\sigma} \cdot \vec{B}-2 i \gamma_{0} \vec{\gamma} \cdot \vec{E}\right) Q,
\end{array}
$$

$q$ being the light spinor and $Q$ the nonrelativistic heavy quark spinor whose upper two components are zero. For axial vector currents, $\Gamma=\gamma_{5} \gamma_{0}$, and for vector currents, $\Gamma=\gamma_{\mu}$. Here $E$ denotes the standard 4-leaf clover electric field.

In addition, we have calculated the matrix elements of the other operators with the right lattice symmetry and up to the mass dimension of $J_{2}$. These are expected to mix under renormalization with the tree level operators. As the renormalization constants (and hence the mixing terms) are currently unknown, we do not discuss them any further. We simulate heavy quarks with masses $a m_{Q}^{0}=1.6,2.0,2.7$ with $n=2 ; 4.0,7.0,10.0$ with $n=1$, and in the static approximation. The three lower mass values are in the region of the $b$ quark, and the others are used to study the extrapolation of the decay constant to the static limit. The preliminary results presented here are based on an ensemble of 57 quenched Wilson configurations, fixed to Landau and then to Coulomb gauge, on a $16^{3} \times 48$ lattice at $\beta=6 / g^{2}=6.0$. To increase statistics we also include the time reversed versions of these configurations. For light and charm quarks we use a tadpole improved clover action. We use two $\kappa$ values $(0.119$ and 0.126$)$ around the charm region and three $(0.1369,0.1375$ and 0.13808 ) around the strange quark mass.

The heavy quark smearing functions are hydrogen-like wave functions with zero or one node, or delta functions. All combinations of
Table 1

Pion masses with degenerate clover quarks.

\begin{tabular}{|l|l|l|l|l|l|}
\hline$\kappa$ & 0.119 & 0.126 & 0.1369 & 0.1375 & 0.13808 \\
\hline$a m_{\pi}$ & $1.526(2)$ & $1.162(2)$ & $0.424(3)$ & $0.363(3)$ & $0.297(4)$ \\
$a m_{\rho}$ & $1.552(2)$ & $1.200(2)$ & $0.554(6)$ & $0.51(1)$ & $0.47(1)$ \\
\hline
\end{tabular}

those smearing functions at source and sink are used. The light quark propagators are always smeared at the source with a Gaussian smearing function with zero or one node, whereas at the sink we use either a smearing identical to that at the source, or a delta function. The results presented here are obtained with the zero-node smearing of the light propagator at the source.

\section{THE SPECTRUM}

\subsection{Light spectroscopy}

In Table 1, we list the masses of pseudoscalar and vector mesons with degenerate clover quarks. These masses are determined from the fall-off of the zero-momentum meson correlator and thus for the heaviest quarks, where one expects significant discretization effects in the dispersion relation, they should only be taken as rough estimates. Using the method described in [i], we find the critical $\kappa$ to be $\kappa_{c}=0.13924(4)$ (in agreement with the UKQCD estimate (5), the value of the isospin averaged light quark kappa $\kappa_{l}=0.13917(4)$, and the strange $\kappa$ to lie between 0.1372 (4) (determined from $K^{*}$ or $\Phi$ mesons), and 0.13755 (19) (from $K$ mesons). The inverse lattice spacing from $m_{\rho}$ is $a^{-1}=1.9(1) \mathrm{GeV}$.

\subsection{Quarkonia}

For each of the heavy quark masses used for the heavy-light simulation, we also calculate the $\mathrm{S}$ and $\mathrm{P}$ wave states of the corresponding heavyheavy mesons, which is computationally quite inexpensive. Results will be presented elsewhere.

\subsection{Heavy-Light: $B$ and $B_{c}$}

For the $B$ and $B_{c}$ spectrum, we study ${ }^{1} S_{0},{ }^{3} S_{1}$, ${ }^{3} P_{0},{ }^{3} P_{1},{ }^{3} P_{2}$ and ${ }^{1} P_{1}$ states. The data on ${ }^{1} P_{1}$ ${ }^{3} P_{1}$ mixing will be reported elsewhere. For the ${ }^{3} P_{2}$ states, operators belonging to both the $E$ and the $T_{2}$ representations of the cubic group are used. 


\subsubsection{Meson masses}

To calculate the mass of the heavy light ${ }^{1} S_{0}$ mesons, we first determine the energy difference $E_{\text {sim }}\left(p^{2}\right)-E_{\text {sim }}(0)$ by fitting the ratio of non-zero and zero momentum meson correlators to an exponential. This is then related to the mass $M_{2}$ by

$E_{\text {sim }}\left(p^{2}\right)-E_{\operatorname{sim}}(0)=\sqrt{p^{2}+M_{2}^{2}}-M_{2}$.

The values for $M_{2}$, determined using $p^{2}=1$ and $p^{2}=2$ (in units of $\left.(2 \pi / 16 a)^{2}\right)$ with $\kappa=0.1369$, are presented in the second and third columns of Table 2. For comparison, the fourth column lists results obtained assuming that the nonrelativistic dispersion relation holds for $p^{2}=1$,

$E_{\text {sim }}\left(p^{2}\right)-E_{\text {sim }}(0)=\frac{p^{2}}{2 M_{2}}$.

Alternatively, the mass shift $\Delta=M_{2}-E_{\text {sim }}$ can be calculated perturbatively. We use $\Delta$ calculated at lowest order in $\alpha_{s}$ and $1 / m_{Q}^{0}$ [6], but for the action used in [3]. $E_{\mathrm{sim}}(0)$ is the rate of exponential fall-off of the zero momentum correlator. These $M_{2}$ are given in the fifth column of Table 2

Additionally, one can try to determine $\Delta$ from the degenerate heavy-heavy $S$ state mesons using

$\Delta=\frac{1}{2} \Delta_{H H}=\frac{1}{2}\left(M_{2}-E_{\text {sim }}\right)^{H H}$.

Determining $M_{2}^{H H}$ with the nonrelativistic dispersion relation (7) for the heavy-heavy mesons, and $E_{\text {sim }}^{H H}$ from the fall-off of the heavy meson correlator, we obtain the last column of Table 2.

We find that all three methods agree for the lighter masses within errors. For the heaviest masses, the deviations are $\sim 10-20 \%$. These are larger than the expected uncertainty of $\sim 0.1$ in $\Delta^{\text {pert }}$ from higher orders in perturbation theory, and indicate the presence of other systematic errors. Thus, in the remainder of this paper, we use $M_{2}$ from the first method.

\subsubsection{Hyperfine splitting}

We extract the hyperfine splitting $\Delta E$ directly from the exponential fall-off of the ratio of the ${ }^{3} S_{1}$ and the ${ }^{1} S_{0}$ correlators. For the three light $\kappa$ values we don't observe any variation of $\Delta E$
Table 2

${ }^{1} S_{0}$ meson masses at $\kappa=0.1369$.

\begin{tabular}{|r|r|l|l|l|l|}
\hline$m_{Q}^{0}$ & \multicolumn{5}{|c|}{$a M_{2}$} \\
& $p^{2}=1$ & $p^{2}=2$ & $N R$ & $m_{Q}^{\text {pert }}$ & $m_{Q}^{H H}$ \\
\hline 1.6 & $2.16(2)$ & $2.13(3)$ & $2.18(2)$ & 2.13 & $2.20(3)$ \\
2.0 & $2.55(2)$ & $2.52(3)$ & $2.56(3)$ & 2.52 & $2.60(5)$ \\
2.7 & $3.23(4)$ & $3.19(5)$ & $3.24(4)$ & 3.22 & $3.33(7)$ \\
4.0 & $4.49(7)$ & $4.40(10)$ & $4.51(8)$ & 4.60 & $4.7(1)$ \\
7.0 & $7.75(11)$ & $7.27(15)$ & $7.79(8)$ & 7.21 & $8.4(2)$ \\
10.0 & $11.0(2)$ & $10.2(3)$ & $11.02(16)$ & 10.06 & $13.1(2)$ \\
\hline
\end{tabular}

Table 3

Hyperfine splitting in lattice units.

\begin{tabular}{|l|l|l|l|}
\hline$a m_{Q}^{0}$ & \multicolumn{2}{|c|}{$O\left(1 /\left(m_{Q}^{0}\right)^{2}\right)$} & $O\left(1 / m_{Q}^{0}\right)$ \\
& $\kappa=0.119$ & $\kappa=0.1369$ & $\kappa=0.137$ \\
\hline 1.6 & $0.0164(3)$ & $0.0198(10)$ & \\
1.71 & & & $0.020(1)$ \\
2.0 & $0.0141(3)$ & $0.0165(9)$ & $0.017(1)$ \\
2.7 & $0.0116(2)$ & $0.0127(7)$ & \\
4.0 & $0.00865(15)$ & $0.0086(6)$ & $0.0091(8)$ \\
7.0 & $0.00548(12)$ & $0.0046(4)$ & \\
8.0 & & & $0.0042(7)$ \\
10.0 & $0.00865(15)$ & $0.0030(4)$ & \\
\hline
\end{tabular}

with $\kappa$, so we present our data without any chiral extrapolation. Even in the charm region, the dependence of $\Delta E$ on $\kappa$ is small. In Table 3, we list the results at $\kappa=0.119$ and $\kappa=0.1369$, as well as the results obtained in [1] with an action correct to $O\left(1 / m_{Q}^{0}\right)$. For the $B$ mesons, we expect the results for the two actions to agree, since the higher order terms in Eq. 3 do not affect the hyperfine splitting. The data, shown in Fig. 1, substantiate this. Thus the predictions for the hyperfine splittings in [1] remain unchanged.

\subsection{3. $P$ states}

A typical effective mass plot for a $P$ wave state with a heavy quark around the $b$ mass and a light quark around the charm mass is shown in Fig. 2 . For lighter $\kappa$ values, the signal is only slightly noisier. The data presented in Table 1 and shown in Fig. 3, indicate that the separations between the $P$ states are qualitatively as expected. Note, however, that as the mixing between ${ }^{1} P_{1}$ and ${ }^{3} P_{1}$ 


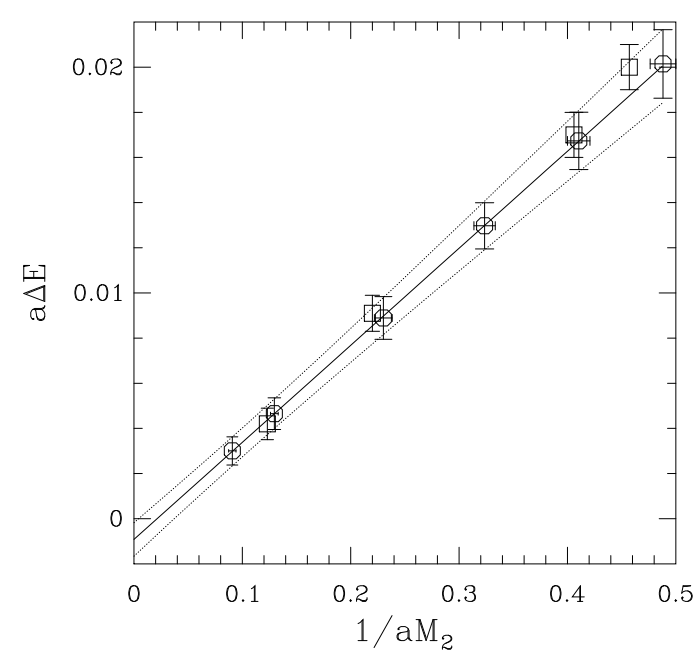

Figure 1. Hyperfine splitting as a function of the mass of the ${ }^{1} S_{0}$ meson presented in column two of Table 2, with a linear fit to the data. Circles are new results, whereas the squares are from [1] using an action correct to only $O\left(1 / m_{Q}^{0}\right)$.

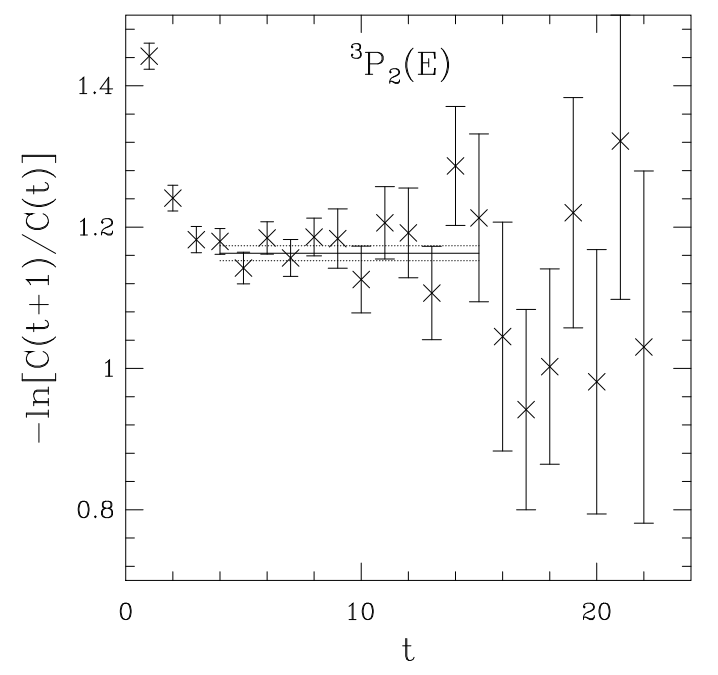

Figure 2. Effective mass of a ${ }^{3} P_{2}$ state with $\kappa=0.119, m_{Q}^{0}=1.6$. Both the light and the heavy quarks use no-node smearing functions at the source, whereas at the sink only the light quark is smeared.
Table 4

$P-{ }^{1} S_{0}$ splittings for $m_{Q}^{0}=1.6, \kappa=0.119$.

\begin{tabular}{|l|c|l|}
\hline state & Lattice Rep. & \multicolumn{1}{|c|}{$\Delta E$} \\
\hline${ }^{3} P_{0}$ & $A_{1}$ & $0.178(13)$ \\
${ }^{1} P_{1}$ & $T_{1}$ & $0.203(12)$ \\
${ }^{3} P_{1}$ & $T_{1}$ & $0.193(8)$ \\
${ }^{3} P_{2}$ & $E$ & $0.204(9)$ \\
${ }^{3} P_{2}$ & $T_{2}$ & $0.200(12)$ \\
\hline
\end{tabular}

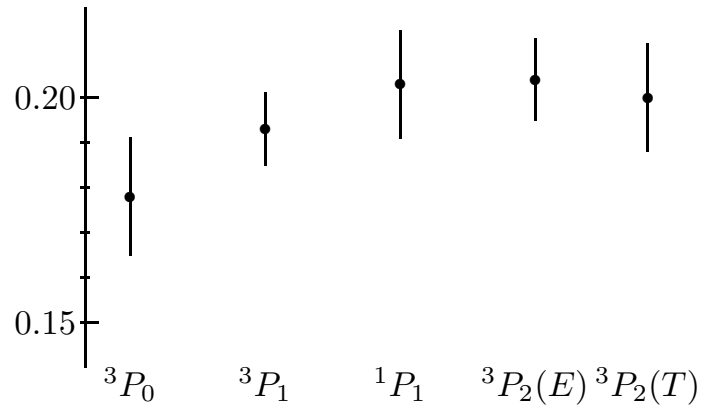

Figure 3. $P-{ }^{1} S_{0}$ splittings lattice units $(\kappa=$ $\left.0.119, m_{Q}^{0}=1.6\right)$. Errors are purely statistical.

has been ignored, thus the ${ }^{1} P_{1}-{ }^{1} S_{0}$ and ${ }^{3} P_{1}-{ }^{1} S_{0}$ splittings are not individually reliable. Also, even though the statistical errors are of the order of the ${ }^{3} P_{2}-{ }^{3} P_{0}$ splitting, a study of the ratio of those correlators indicates that both lattice representations of the ${ }^{3} P_{2}$ are equal and heavier than the ${ }^{3} P_{0}$.

\section{DECAY CONSTANTS}

\subsection{Analysis}

The decay constants are related to the one meson production amplitudes of the local pseudoscalar or vector current $J_{L}$.

$f \sqrt{M}=\sqrt{2} A_{L}=\sqrt{2 / M}\left\langle 0\left|J_{L}\right| B\right\rangle$.

To determine $A_{L}$, we fit to the smeared-smeared and smeared-local ${ }^{1} S_{0}$ and ${ }^{3} S_{1}$ correlators with different operator and smearing combinations at the source and sink to the exponential form:

$\left\langle O_{s n k} O_{s r c}\right\rangle=A_{O_{s r c}, O_{s n k}} \exp \left(-E_{s i m}^{s r c, s n k} t\right)$. 
The measured matrix of the amplitudes is then factorized as $A_{O_{s r c}, O_{s n k}}=A_{O_{s r c}} A_{O_{s n k}}$ by minimizing the overall $\chi^{2}$. The matrix element for the improved current, specified in Eq. 5, is then constructed by simply adding the various terms. To get the physical decay constants we extrapolate linearly in $1 /(2 \kappa)$ to $\kappa=\kappa_{l}$ and $\kappa=\kappa_{s}$. The entire procedure is carried out in a singleelimination jackknife loop to estimate the errors in the final quantities.

The signal for the effective amplitudes of the correlators show clear plateaus except for the static case. In Fig. 4, we show an example of the effective amplitude of the largest $O\left(1 /\left(m_{Q}^{0}\right)^{2}\right)$ current correction to the ${ }^{1} S_{0}$ decay constant.

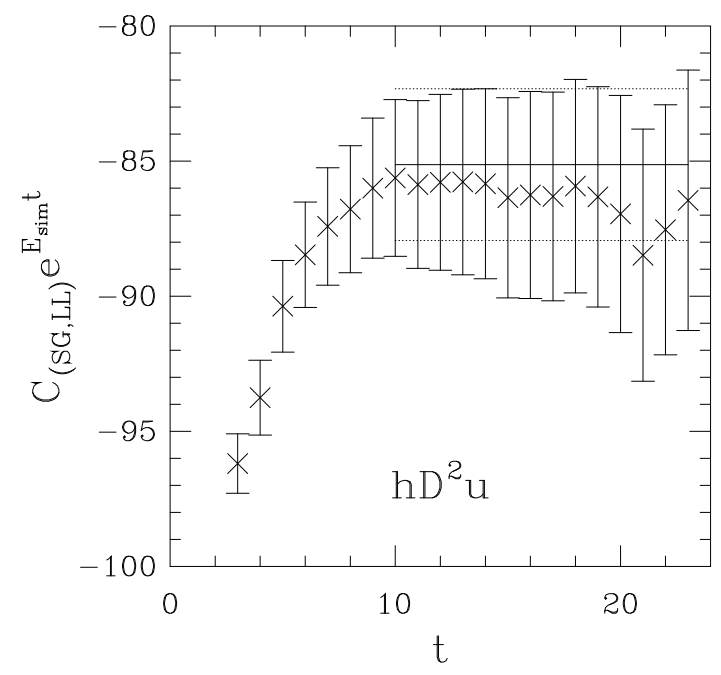

Figure 4. Effective amplitude for the correlator of a ${ }^{1} S_{0}$ current correction at $m_{Q}^{0}=1.6$ and $\kappa=$ 0.1369 . Both the quarks use smearing functions with no nodes at the source, and are unsmeared at the sink.

\subsection{Results}

We present a study of the effect of improving the action and the operators to $O\left(1 /\left(m_{Q}^{0}\right)^{2}\right)$. It is important to recall that all coefficients have
Table 5

The contributions to the decay constants from $O\left(1 / m_{Q}^{0}\right)$ current correction (second column) and the three $O\left(1 /\left(m_{Q}^{0}\right)^{2}\right)$ current corrections for $\kappa=$ 0.1369 . All numbers in the table have been multiplied by 100 for convenience.

\begin{tabular}{|l|l|l|l|l|}
\hline$m_{Q}^{0}$ & $\frac{\sigma \cdot D}{2 m_{Q}^{0}}$ & $\frac{\sigma \cdot B}{8\left(m_{Q}^{0}\right)^{2}}$ & $\frac{\sigma \cdot E}{4\left(m_{Q}^{0}\right)^{2}}$ & $\frac{D^{2}}{8\left(m_{Q}^{0}\right)^{2}}$ \\
\hline \multicolumn{5}{|c|}{$\kappa=0.1369$} \\
\hline 1.6 & $-5.40(19)$ & $0.82(04)$ & $1.16(05)$ & $-1.23(04)$ \\
2.0 & $-4.59(17)$ & $0.49(03)$ & $0.75(03)$ & $-0.86(03)$ \\
2.7 & $-3.59(14)$ & $0.25(02)$ & $0.41(02)$ & $-0.53(02)$ \\
4.0 & $-2.62(12)$ & $0.10(01)$ & $0.19(01)$ & $-0.27(01)$ \\
7.0 & $-1.97(07)$ & $0.035(3)$ & $0.060(5)$ & $-0.134(5)$ \\
10.0 & $-1.58(06)$ & $0.018(1)$ & $0.031(3)$ & $-0.080(3)$ \\
\hline \multicolumn{5}{|c|}{$\kappa=0.119$} \\
\hline 1.6 & $-8.10(17)$ & $1.28(03)$ & $1.60(03)$ & $-3.27(07)$ \\
2.0 & $-7.18(16)$ & $0.78(02)$ & $1.07(02)$ & $-2.44(05)$ \\
2.7 & $-6.00(16)$ & $0.41(01)$ & $0.62(02)$ & $-1.61(04)$ \\
4.0 & $-4.67(14)$ & $0.18(01)$ & $0.30(01)$ & $-0.90(02)$ \\
7.0 & $-3.04(08)$ & $0.055(2)$ & $0.106(3)$ & $-0.39(01)$ \\
10.0 & $-2.28(07)$ & $0.025(1)$ & $0.054(2)$ & $-0.231(7)$ \\
\hline
\end{tabular}

tadpole-improved tree-level values with no perturbative corrections. Thus, the following results should be considered qualitative and preliminary.

The data in Table 5 show that in the mass region of the $B$ meson, $\left(m_{Q}^{0} \sim 2.0\right)$, the individual current corrections arising at $O\left(1 /\left(m_{Q}^{0}\right)^{2}\right)$ are $\sim 20 \%$ of the $O\left(1 / m_{Q}^{0}\right)$ corrections. However for the pseudoscalar decay constant the three $O\left(1 /\left(m_{Q}^{0}\right)^{2}\right)$ terms add up to a result close to zero because they have different signs. (For the vector decay constant these three terms come with the same sign). The data for the different heavy quarks is shown in Fig. 旬.

The effects of the $O\left(1 /\left(m_{Q}^{0}\right)^{2}\right)$ corrections in the hamiltonian are shown in Fig. 6 where we compare the chirally extrapolated results against the $O\left(1 / m_{Q}^{0}\right)$ estimates given in [1]. We find that the $O\left(1 /\left(m_{Q}^{0}\right)^{2}\right)$ data indicates a smaller slope, though the difference is marginally significant. We are in the process of calculating the perturbative corrections to the coefficients in order to extract the physical value of the decay matrix el- 


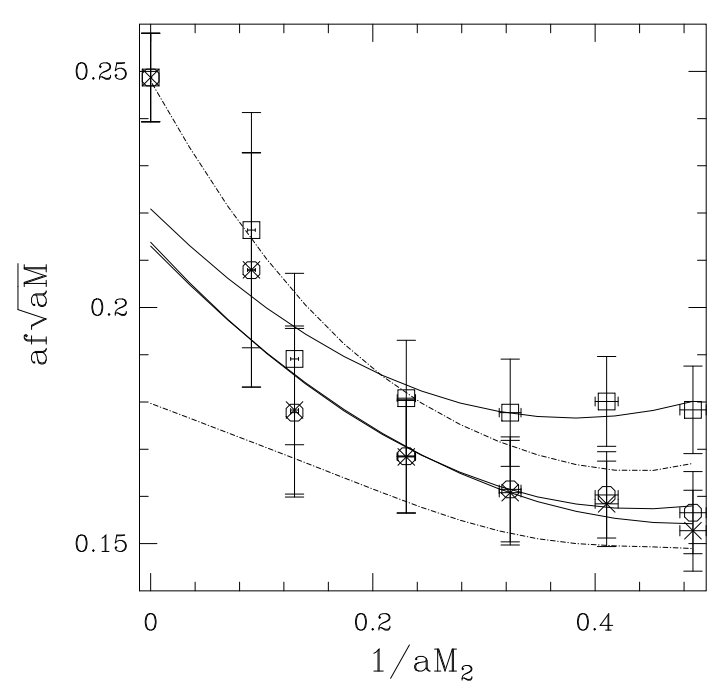

Figure 5. Comparison of the chirally extrapolated values of $f \sqrt{M}$ with various current corrections: circles include all corrections through $O\left(1 /\left(m_{Q}^{0}\right)^{2}\right)$, crosses include up to $O\left(1 / m_{Q}^{0}\right)$ corrections, whereas squares are the leading order results. The fits shown are uncorrelated quadratic fits: broken lines show the error on the fit to the circles.

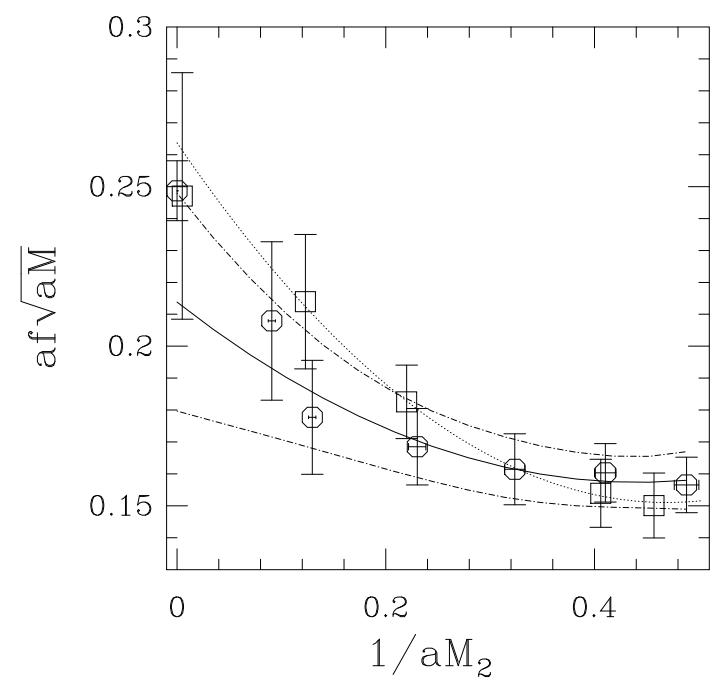

Figure 6 . The chirally extrapolated $f \sqrt{M}$ calculated consistently at $O\left(1 / m_{Q}^{0}\right)$ from [1] (data: squares, fit: dotted line) and at $O\left(1 /\left(m_{Q}^{0}\right)^{2}\right)$ (data: circles, fit: solid line). The error in the $O\left(1 /\left(m_{Q}^{0}\right)^{2}\right)$ points is shown by a dashed line.
Table 6

$(f \sqrt{M})_{P S}$ at various levels of the current corrections (no corrections, correct at $O\left(1 / m_{Q}^{0}\right)$ and at $\left.O\left(1 /\left(m_{Q}^{0}\right)^{2}\right)\right)$. The last column shows results of a calculation where both the Hamiltonian and current are correct to only $O\left(1 / m_{Q}^{0}\right)$.

\begin{tabular}{|l|l|l|l|l|}
\hline$m_{Q}^{0}$ & \multicolumn{1}{|c|}{$O(1)$} & $O\left(\left(m_{Q}^{0}\right)^{-1}\right)$ & $O\left(\left(m_{Q}^{0}\right)^{-2}\right)$ & [1] \\
\hline 1.6 & $0.178(09)$ & $0.153(9)$ & $0.157(9)$ & \\
1.71 & & & & $0.150(11)$ \\
2.0 & $0.180(10)$ & $0.158(9)$ & $0.160(9)$ & $0.154(12)$ \\
2.7 & $0.178(11)$ & $0.161(11)$ & $0.161(11)$ & \\
4.0 & $0.181(12)$ & $0.169(12)$ & $0.168(12)$ & $0.182(13)$ \\
7.0 & $0.189(18)$ & $0.178(18)$ & $0.178(18)$ & \\
8.0 & & & & $0.21(2)$ \\
10.0 & $0.216(25)$ & $0.208(24)$ & $0.208(25)$ & \\
$\infty$ & $0.249(9)$ & $0.249(9)$ & $0.249(9)$ & $0.25(4)$ \\
\hline
\end{tabular}

ements and their slope as a function of $1 / M$.

\section{Acknowledgements}

This work was supported by SHEFC, the U.S. DOE, PPARC, and the NATO under grant number CRG 941259. We acknowledge the ACL at LANL and NCSA at Urbana for computational support.

\section{REFERENCES}

1. A. Ali Khan et al., Nucl. Phys. B (Proc. Suppl.) 47 (1996) 425.

2. S. Collins, these proceedings.

3. C.T.H. Davies et al., Phys. Rev D 50 (1994) 6963 .

4. T. Bhattacharya et al., Phys. Rev. D 53 (1996) 6486.

5. R. Kenway, these proceedings.

6. C.J. Morningstar, Phys. Rev D 50 (1994) 5902 and private communication.

7. C.T.H. Davies et al., hep-lat/9602020. 